Revue de droit comparé du travail et de la sécurité sociale

\title{
La notion de travailleur subordonné en Espagne face aux nouvelles formes d'emploi
}

Jesús Cruz Villalón

\section{(2) OpenEdition}

1 Journals

\section{Édition électronique}

URL : https://journals.openedition.org/rdctss/1554

DOI : 10.4000/rdctss. 1554

ISSN : 2262-9815

Éditeur

Centre de droit comparé du travail et de la sécurité sociale

\section{Édition imprimée}

Date de publication : 1 juin 2019

Pagination : 48-59

ISSN : 2117-4350

\section{Référence électronique}

Jesús Cruz Villalón, « La notion de travailleur subordonné en Espagne face aux nouvelles formes d'emploi », Revue de droit comparé du travail et de la sécurité sociale [En ligne], 2 | 2019, mis en ligne le 01 novembre 2021, consulté le 11 novembre 2021. URL : http://journals.openedition.org/rdctss/1554 ; DOI : https://doi.org/10.4000/rdctss. 1554

\section{cc)}

Revue de droit comparé du travail et de la sécurité sociale est mise à disposition selon les termes de la Licence Creative Commons Attribution - Pas d'Utilisation Commerciale - Pas de Modification 4.0 International. 


\section{LA NOTION DE TRAVAILLEUR SUBORDONNÉ EN ESPAGNE FACE AUX NOUVELLES FORMES D'EMPLOI ${ }^{1}$}

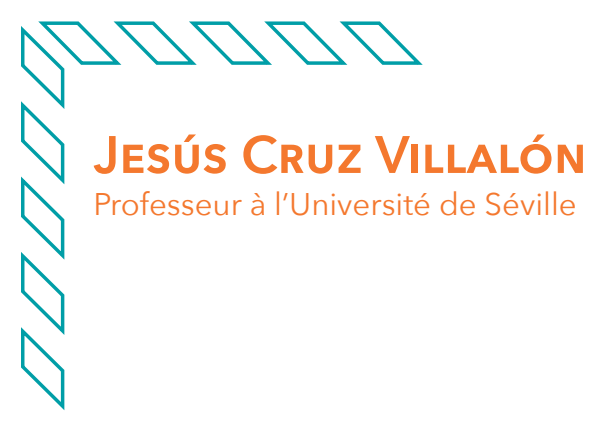

L

a délimitation des frontières entre, d'une part le travail subordonné soumis à la législation du travail et, d'autre part, le travail indépendant soumis au droit civil, commercial ou administratif, est une question sensible depuis l'origine du droit du travail. Selon les écarts notables induits par telle ou telle forme d'activité en termes de conditions professionnelles et, surtout de coûts économiques pour les entreprises, des tensions apparaissent quant au choix de l'encadrement juridique privilégié, notamment s'agissant de leur réglementation ou non par la législation du travail. Les intérêts en jeu sont décisifs. Or, ni l'amélioration des textes juridiques dans la délimitation conceptuelle des exigences requises pour qu'une prestation de service soit soumise ou non à la législation du travail, ni les efforts consentis par la justice pour appliquer les principes interprétatifs cohérents à cet égard, n'ont permis à ce jour d'apaiser les divergences entre les parties; de nouvelles réalités en matière d'emploi surviennent sans arrêt, provoquant la résurgence des vieux débats².

1 Ce travail est un résumé de celui qui a été publié dans la Revista de derecho social $n^{\circ} 83$ (2018), sous le titre « La notion de travailleur subordonné face aux nouvelles formes de travail ».

2 Por todos, AA. W., Las fronteras del Derecho del Trabajo en el marco comparado europeo: autónomos y becarios, XXVI Congreso Nacional de Derecho del Trabajo y de la Seguridad Social, Cinca, Madrid, 2016 ; S. González Ortega, « Trabajo asalariado y trabajo autónomo en las actividades profesionales a través de plataformas informáticas», Temas Laborales, 2017, 138, p. 85 ; M. R. Martínez Barroso, «Trabajo autónomo y trabajo subordinado. Delimitación. Análisis y propuestas de reforma », Estudios Financieros 273, 2007 ; A. Todoli, « Nuevos indicios de laboralidad como resultado de las nuevas empresas digitales, in M. Rodríguez Piñero et M. Hernández Bejarano, Economía colaborativa y trabajo en plataformas: realidades y desafíos, Bomarzo, Albacete, 2017, p. 223. 
Ces nouveautés s'expliquent par deux facteurs interdépendants. D’une part, certains changements technologiques conduisent à minimiser l'importance de données factuelles, traditionnellement considérées comme permettant d'identifier les aspects juridiques les plus emblématiques de la subordination. D'autre part, la mise en œuvre de changements dans l'organisation de l'entreprise donne lieu à des formes de décentralisation productive, réelle ou apparente, par le biais de mécanismes de recrutement non contractuels, qui suscitent des doutes sur le bien-fondé ou non de telles pratiques.

Le débat actuel sur les changements technologiques tend à se concentrer sur l'impact des plateformes numériques, ce qui traduit une vision très réductrice de la réalité. En effet, ces évolutions ne concernent pas exclusivement la numérisation, d'autres transformations technologiques sont à l'œuvre et passent inaperçues alors qu'elles devraient être prises en considération. Par ailleurs, bien souvent d'autres facteurs d'ordre organisationnel ou $d^{\prime}$ ' ingénierie contractuelle » sont tout aussi influents, si ce n'est plus, au regard de la thématique que nous traitons.

Aujourd'hui, la première transformation notable tient au dépassement du modèle de production fordiste, caractérisé par une main-d'œuvre présente au sein même des locaux de l'entreprise et travaillant de façon concomitante. Au-delà des impératifs légaux, l'entreprise fordiste, pour des raisons techniques et de productivité, devait nécessairement embaucher ses salariés dans un cadre juridique soumis à la législation du travail, les missions pour lesquelles l'entreprise pouvait choisir d'agir en dehors des normes du travail étant très rares. Lorsque le fordisme disparaît, les entreprises vont alors disposer d'une capacité organisationnelle et technologique qui leur permet de recourir à certains services, sans passer par les formes classiques de subordination du travailleur, tout en maintenant un contrôle identique - voire plus intense - sur la manière dont le travail est effectué et sur les résultats obtenus. En apparence, le travailleur dispose d'une large autonomie de gestion et de décision mais, dans les faits, l'entreprise détient des instruments clés qui donnent lieu aux formes de subordination du travail les plus orthodoxes.

Divers points de vue laissent entendre que nous allons vers un scénario de très forte augmentation du travail autonome et, parallèlement, vers un processus de déclin du travail subordonné. Les seules données objectives dont nous disposons sont les statistiques issues de l'Enquête sur la population active (Encuesta de Población activa) qui, jusqu'à présent, contredisent ces pronostics. Les principaux chiffres montrent que depuis plusieurs décennies, le taux de salarisation est supérieur à $80 \%$, alors que celui des travailleurs indépendants au sens strict du terme (aucun salarié à leur service) est inférieur à $12 \%$, sans que ces pourcentages évoluent de manière substantielle. A l'inverse, les statistiques les plus fiables révèlent généralement que les changements techniques, organisationnels et productifs, ne se traduisent pas par un recul du travail subordonné sur notre marché du travail, ni, par conséquent, par une augmentation du travail indépendant. Toutefois, si nous ne sommes pas actuellement confrontés à une évolution quantitative de la place du travail salarié sur le marché du travail, il n'en demeure pas moins que le débat traditionnel sur les frontières entre le travail subordonné et le travail indépendant conserve toute son importance et que l'issue de ce débat qualitatif pourrait finir par avoir une incidence sur le quantitatif.

En termes de contentieux, le nombre de procès dans lesquels il est débattu de la nature de la relation de travail est restreint, et rien n'indique qu'il ait augmenté ces dernières années, en dépit d'un énorme impact sur le débat doctrinal, surtout au sein des médias. 
Jusqu'à présent, il n'existe pas de jurisprudence concluante à ce sujet, car aucune décision de la Cour suprême n'a encore été rendue sur les questions les plus controversées liées à l'utilisation des plateformes de travail. Seule la Cour de justice des Communautés européennes au Luxembourg s'est prononcée concernant les agissements d'Uber Espagne : la plateforme numérique faisait valoir qu'elle n'était qu'un simple intermédiaire entre utilisateurs et fournisseurs de services, mais la Cour de Luxembourg a considéré que son activité allait plus loin et a qualifié Uber de société fournissant un service de transport de passagers. Toutefois, sans se prononcer sur la question de savoir si cette prestation avait été effectuée par des professionnels indépendants ou par l'intermédiaire de salariés au service d'Uber - bien qu'en l'espèce, certaines idées puissent sembler implicites dans l'arrêt de la Cour - il ne s'agissait pas de la question posée à titre préjudiciel ${ }^{3}$.

Dans les juridictions nationales, il y a eu des prises de position disparates principalement au sujet de distributeurs de marchandises répondant à des commandes passées via des plateformes numériques. Dans deux cas, le caractère de travail subordonné entrant dans le champ d'application de la législation du travail ${ }^{4}$ a été reconnu, tandis que dans deux autres, les distributeurs ont été qualifiés de travailleurs autonomes économiquement dépendants, n'entrant pas dans le champ d'application de la législation du travail ${ }^{5}$. Quoi qu'il en soit, ces décisions rendues par des instances judiciaires de rang inférieur ont fait l'objet d'un appel et sont donc en attente du jugement qui sera prononcé, le cas échéant, par le Tribunal suprême.

Malgré ce qui précède, et au-delà du travail sur les plateformes, le Tribunal suprême a rendu d'importants arrêts faisant jurisprudence, portant sur la nature de la relation de travail induite par certaines formes d'emploi adaptées à des besoins précis et inhérents à l'organisation du travail. Cette jurisprudence fournit des indices importants, qui devraient nous renseigner sur les critères retenus par le Tribunal face à ces nouvelles réalités et nous servir de lignes directrices pour la construction de catégories générales.

\section{I - L'ABSENCE DE CHANGEMENTS SUBSTANTIELS}

\section{A - L'ABSENCE DE CHANGEMENTS LÉGISLATIFS SUBSTANTIELS}

Malgré le débat persistant sur les frontières entre travail indépendant et travail subordonné, en dépit des changements organisationnels et de l'émergence de nouvelles formes d'emploi, le législateur est resté fidèle à la définition du travail et aux exigences juridiques traditionnelles du travail subordonné prévues par la législation du travail. Face aux multiples modifications apportées au texte du Statut des travailleurs depuis sa première version en 1980, peu de changements - qui plus est des changements secondaires - ont été apportés aux articles clés portant sur la frontière entre travail subordonné et travail indépendant (articles 1 et 2 du Statut des travailleurs). Les exigences classiques liées à la prestation de services à titre personnel sont maintenues : travail volontaire, rémunéré,

3 STJUE 20 décembre 2017, C-434/15, Affaire Uber Systems Spain, ECLI:EU:C:2017:981. Dans des termes similaires, STJUE 10 avril 2018, C-320/16, affaire Uber France SAS, ECLI:EU:C:2018:221.

4 Décision du Tribunal Social de Valence n 6, 1er juin 2018, ECLI:EN:JSO:2018:1482. Décision du Tribunal Social de Madrid n 33, 11 février 2019, autos 1214/2018, JUR 2019\48639.

5 Décision du Tribunal social de Madrid n³9, 3 septembre 2018, ECLI : ES:JSO:2018:3042. Décision du Tribunal Social de Madrid n 17 du 11 janvier 2019, ordonnances 418/18. 
dépendant et pour le compte d'autrui. De plus, lorsque le Statut du travail autonome (Ley del Estatuto del Trabajo Autónomo) a été approuvé en 2007, il en a donné une définition qui semble n'être que l'inverse des dispositions énoncées dans le Statut des travailleurs (article 1.1 LETA).

Ce n'est que dans certains cas particuliers que des modifications législatives importantes ont été apportées, telles l'exclusion du travail de certains transporteurs dans le cadre de la réforme de 1994, ou la prise en compte de certaines relations de travail particulières qui, en pratique, intégrait dans le champ d'application de la législation du travail des cas de figure jusqu'alors exclus ou pour lesquels il existait des réserves.

\section{B - L'ABSENCE D'ÉVOLUTION SUBSTANTIELLE DES CRITÈRES DE LA JURISPRUDENCE}

La jurisprudence, elle aussi, a globalement évolué de façon linéaire dans l'interprétation de la loi. Face aux nouvelles formes d'emploi, les juridictions compétentes en droit du travail ont adopté une approche qu'elles ont elles-mêmes qualifié de « continuité argumentative ».

\section{1 - Qualification formelle par les parties et analyse matérielle de la relation de travail}

Le postulat de départ est que l'instance judiciaire doit procéder à la vérification de la réalité matérielle du type de relation professionnelle existant entre les parties, par-delà le formalisme affiché. Dès lors, la principale conséquence négative tient au fait que la qualification juridique attribuée par les parties au contrat qui les lie n'est pas pertinente ${ }^{6}$, ou que d'autres données formelles sont sans influence - comme celles relatives à l'encadrement de la sécurité sociale ou au respect de certaines obligations fiscales- pas plus qu'il n'est accordé d'importance au fait que le salarié puisse avoir un statut d'associé d'une société7. Il est arrivé qu'un vote au sein du Tribunal défende la position selon laquelle lorsqu'il existe une "zone grise » et une certaine autonomie dans l'exécution de la prestation de travail, la volonté exprimée par les parties doit primer s'agissant de la détermination des droits et obligations inhérents à la relation contractuelle ${ }^{8}$. Il s'agit là d'une tentative isolée qui a été défendue, mais qui n'a pas été retenue par le vote majoritaire et n'est pas réapparue dans le débat judiciaire.

En outre, il convient de garder à l'esprit que le postulat de départ énoncé plus haut est fondé sur la présomption selon laquelle, en règle générale, la qualification du contrat est imposée au travailleur par l'entreprise, celle-ci asseyant, dès le départ, sa supériorité vis-à-vis du prestataire de services. Par conséquent, cette vérification du critère de la réalité matérielle du type de relation existant entre les parties n'a lieu que dans les cas de figure où le contrat n'est pas qualifié de contrat de travail. Autrement dit, le critère $a$, sans que cela soit explicite, un caractère unidirectionnel. Inversement, puisqu'elle n'est pas bidirectionnelle, la signature d'un contrat qualifié de contrat de travail doit donner lieu à la présomption selon laquelle ce lien contractuel est précisément la nature de la relation qui lie les parties, d'où l'importance du titre donné par celles-ci au contrat lorsqu'il est qualifié de contrat de travail.

6 STS (Décision du Tribunal Suprême) du 16 novembre 2017, rec. 2806/2015, ECLI:ES:TS:2017:4552.

7 STS du 25 mars 2013, rec. 1564/2012, ECLI:ES:TS:2013:2389.

8 STS du 19 février 2014, rec. 3205/2012, ECLI:ES:TS:2014/1404. 
Jusqu'à présent, le seul cas dans lequel la jurisprudence avait accordé une certaine importance à une donnée formelle pour exclure la dimension contractuelle de la relation de travail concernait le statut de membre d'une coopérative. Dans cette affaire, où l'on partait du principe qu'il n'y avait pas de dépendance vis-à-vis d'autrui puisque ce sont les membres qui perçoivent les recettes de la coopérative, on considérait que le demandeur - donc le prestataire de services - devait fournir une preuve rigoureuse de l'existence d'un acte frauduleux au regard de la loi. Inversement, les données formelles liées au statut de membre de la coopérative ont été prises en compte et considérées comme pertinentes?. Cependant, un autre arrêt récemment rendu revient sur le critère précédent. Partant de l'existence éventuelle de pratiques frauduleuses dans I'utilisation du régime coopératif, il est considéré au vu de la concordance de certains indices en ce sens, que « la coopérative a la charge de prouver qu'elle exerce une véritable activité au profit de ses membres $»^{10}$.

\section{2 - La prestation de services comme présupposée quasi-évidente}

Un exemple tout à fait exceptionnel fait état d'un cas très frappant où il n'y avait même pas eu de prestation de services. La décision rendue considère que c'est l'entreprise ellemême qui reconnaît le statut de salariés à ceux qui se sont contentés d'exercer des activités de représentation syndicale sans travailler. En effet, pendant toute la durée de la relation avec les travailleurs, l'entreprise a effectué le paiement des salaires dans le cadre de contrats qualifiés de contrats de travail, ce qui équivaut à une manifestation explicite de la volonté d'être lié comme employeur ${ }^{11}$. En se basant sur les faits eux-mêmes, cet arrêt affirme que la signature des contrats de travail met en évidence la nature de la relation de travail.

\section{3 - La non pertinence de la présomption de la relation de travail}

La jurisprudence a défendu, de façon récurrente, l'existence dans notre système juridique de la présomption de la relation de travail, en vertu d'une disposition juridique précise (article 8, paragraphe 1 du Statut des travailleurs). Cependant, le fondement légal d'une telle présomption est plus que discutable. Le principe est que la présence de certains éléments factuels (travail rémunéré, dépendant et pour le compte d'autrui) suffit à prouver la relation contractuelle, sans qu'il soit nécessaire de démontrer l'existence d'un contrat écrit ou oral, le simple comportement probant pouvant même suffire. Pour preuve, bien que notre jurisprudence appelle régulièrement à la présomption de la relation de travail, elle ne va pas jusqu'à en préciser la portée concrète ${ }^{12}$. Dans certains cas, on parle même de la présence d'une présomption «ténue » de la relation de travail ${ }^{13}$.

La ligne de conduite observée est d'ailleurs plutôt celle d'une présomption d'absence de relation de travail, à partir du constat de certains éléments factuels. C'est précisément ce que fait le législateur, en 1994, lorsqu'il exclut de la relation de travail les titulaires de la carte administrative de transporteur utilisant pour leurs prestations de service des véhicules

9 STS du 17 décembre 2001, rec. 244/2001.

10 STS du 18 mai 2018, rec. 3513/2016, ECLI:ES:TS:2018:2263.

11 STS du 12 avril 2018, rec. 119/2016, ECLI:ES:TS:2018:1776.

12 STS du 16 novembre 2017, rec. 2806/2015, ECLI:ES:TS:2017:4552; STS du 25 mars 2013, rec. 1564/2012, ECLI:ES:TS:2013:2389; STS du 29 novembre 2010, rec. 253/2010, ECLI:ES:TS:2010:7764; STS du 20 juillet 2010, rec. 3344/2009, ECLI:ES:TS:2010:4450; STS du 7 octobre 2009, rec. 4160/2008, ECLI:ES:TS:2009:6415.

13 STS du 8 février 2018, rec. 3389/2015, ECLI:ES:TS:2018:589. 
utilitaires dont ils détiennent la propriété ou la pleine jouissance (article 1, paragraphe 3, point g, du Statut des travailleurs) ${ }^{14}$.

\section{4 - La prestation de services comme contrat générique: travail pour le compte d'autrui et subordination comme critères supplémentaires de la relation de travail}

Récemment, la jurisprudence a souligné que la prestation de services soumise au droit civil constituait une modalité de contrat générique par rapport au contrat de travail soumis à la législation du travail, dans la mesure où le contrat de travail n'était qu'une sous-catégorie ou une variante de la première. Les caractéristiques de la prestation de services sont toutes manifestes dans le contrat de travail, mais ce dernier présente en plus des spécificités qui lui sont propres. Cette précision permet à la jurisprudence de justifier les difficultés qu'elle rencontre pour délimiter les frontières entre les deux régimes juridiques; elle sert à expliquer que toute la complexité vient du fait que seuls certains éléments spécifiques de la prestation de services permettent de privilégier la législation du travail ${ }^{15}$.

En particulier, pour la jurisprudence, ce que le contrat de travail a - et que la prestation de services n'a pas - tient à la présence cumulative du travail pour le compte d'autrui et de la dépendance. L'absence de l'un ou l'autre d'entre eux se traduit par leur exclusion du champ d'application de la législation du travail, alors qu'il semblerait que l'absence des deux nous placerait dans la catégorie des travailleurs indépendants. Ce qui précède semble suggérer l'idée, difficile à accepter, que le travail pour le compte d'autrui et la subordination vont nécessairement de pair.

Toutefois, il n'en est pas vraiment ainsi. Si les deux critères sont officiellement requis, la question se pose de savoir s'il n'existe pas un certain vide juridique pour traiter des situations où seul l'un d'entre eux est présent. D'après une lecture juridique systématique et compte tenu de l'interprétation jurisprudentielle, lorsque l'un des deux critères fait défaut, nous ne nous trouvons pas dans le champ d'application du Statut des travailleurs mais dans celui du Statut du travail autonome.

II existe des cas dans lesquels le travail indépendant peut être réalisé, même si la prestation de services est effectuée dans le cadre d'un régime de dépendance au sein de l'organisation collective pour laquelle on travaille : c'est le cas des membres des coopératives de travail associé ${ }^{16}$. Inversement, il existe aussi un travail autonome lorsque celui-ci est effectué pour le compte d'autrui mais sans subordination. Les nouvelles formes d'organisation du travail, en particulier les processus de décentralisation productive, font qu'un travailleur indépendant peut être amené à exécuter son service en toute indépendance du point de vue organisationnel, mais pour le compte de l'entreprise principale qui sous-traite un service. II ne s'agirait en aucun cas d'un faux indépendant, dans la mesure où il est établi que le travail n'est pas soumis à un régime de subordination organisationnelle et qu'il n'y a donc pas de dépendance dans le travail accompli pour le compte d'autrui. Ces situations de décentralisation productive, où un travail autonome

14 STS du 18 mai 2018, rec. 3513/2016, ECLI:ES:TS:2018:2263.

15 STS du 8 février 2018, rec. 3389/2015, ECLI:ES:TS:2018:589; STS du 24 janvier 2018, rec. 3394/2015, ECLI:ES:TS:2018:608; STS du 16 novembre 2017, rec. 2806/2015, ECLI:ES:TS:2017:4552; STS du 3 novembre 2014, rec. 739/2013, ECLI:ES:TS:2014:5118; STS du 29 de novembre 2010, rec. 253/2010, ECLI:ES:TS:2010:7764.

16 Implicitement, STS du 17 décembre 2001, rec. 244/2001. 
est effectué pour le compte d'autrui mais sans subordination, sont celles qui, aujourd'hui, augmentent la difficulté à délimiter les frontières entre les deux régimes juridiques.

\section{5 - Le recours au salaire garanti, source de confusion}

La jurisprudence fait parfois allusion à l'exigence de rémunération prévue par le Statut des travailleurs. La situation est claire lorsque nous sommes dans le cadre d'un travail effectué à titre bénévole ou d'un travail purement amateur, dont il ne découle aucun droit à rémunération. L'activité sera même qualifiée de non-professionnelle lorsque des sommes sont perçues à titre de compensation uniquement, pour des dépenses encourues mais non pour l'exécution d'un travail ${ }^{17}$. L'élément le plus important du cas de figure en question tient au fait que la rémunération, plutôt qu'une exigence " en soi », s'explique par le fait d'avoir précédemment qualifié la situation de prestation de services subordonnée. D'autres décisions judiciaires affirment la nécessité pour le travailleur de bénéficier, en plus, d'une "rémunération garantie ${ }^{18}$. Il ne fait aucun doute que l'une des différences entre le travail indépendant et le travail subordonné réside, pour ce dernier, dans l'absence de risque concernant la rémunération : la perception du salaire du travailleur salarié est garantie, quel que soit le résultat du travail fourni, alors que pour le travailleur indépendant la rémunération se fait en fonction des bénéfices obtenus et dépend donc de la bonne ou de la mauvaise gestion de son entreprise. Cependant, on constate que cet élément de la rémunération "garantie » perd aujourd'hui progressivement de sa pertinence, compte tenu des transformations qui s'opèrent sur le marché du travail et dans les nouvelles formes d'emploi. D'une part, dans certains types de professions à responsabilité ou à forte dimension technique, la rémunération par objectifs est de plus en plus importante. Dans ces situations, même si la rémunération « garantie » est très faible, ce type de prestations n'en demeure pas moins soumis à un système de contrats de travail. On pourrait même citer des cas inverses où, à la suite de prestations effectuées par un travailleur autonome dans le cadre de la décentralisation productive, l'entreprise cliente établit une relation de continuité avec le travailleur indépendant, en prenant certains engagements de loyauté mutuelle renforcée par la reconnaissance d'honoraires minimaux " garantis » en toutes circonstances.

\section{II - UNE RELECTURE SOUPLE DE LA SUBORDINATION}

Fort de ce qui précède, le critère de subordination conserve toute sa vitalité et jouit d'une bonne santé juridique en tant qu'instrument clé et utile pour marquer la frontière entre travail indépendant et travail salarié. Ceci nous amène aussi à reconnaître que les changements organisationnels et technologiques au sein des entreprises, tout comme les nouvelles méthodes de travail, exigent une nouvelle lecture de la présence - dans chaque cas spécifique - de l'exigence de subordination.

Depuis plusieurs décennies, notre jurisprudence a adopté une position très souple à l'égard des éléments factuels devant être réunis pour qualifier un service rendu comme s'inscrivant dans une relation de subordination. II y a longtemps que la subordination a cessé d'être rattachée au travail industriel dans la chaîne de production fordiste.

17 STS du 7 novembre de 2017, rec. 3573/2015, ECLI:ES:TS:2017:4087.

18 STS du 19 février 2014, rec. 3205/2012, ECLI:ES:TS:2014:1404; STS du 8 février 2018, rec. 3389/2015, ECLI:ES:TS:2018589. 
L'incorporation de travailleurs d'un certain niveau technique a conduit à une importante marge d'autonomie dans l'exécution du travail, sans que la qualification de subordination du travail effectué ne disparaisse. À tel point que la jurisprudence a été amenée à affirmer que, pour les travaux exigeant un haut niveau de professionnalisme technique, la notion de dépendance est assouplie et " peut même disparaître complètement " au regard des exigences déontologiques et professionnelles d'indépendance technique qui caractérisent leur exercice. Ce raisonnement poussé à l'extrême a conduit à qualifier de relation de travail le lien entre certains dentistes et la clinique où ils exerçaient, malgré la présence d'éléments conduisant traditionnellement à qualifier l'activité d'indépendante. Les juges ont notamment mis en exergue l'exécution « intuitu personae » de la prestation de service, le travail pour le compte d'autrui, le paiement du salaire par l'employeur, la mise à disposition du travail au sein de l'organisation et de la direction de la clinique, le conditionnement de la journée aux heures d'ouverture au public, le mécanisme de paiement de la rémunération ${ }^{19}$. Un positionnement similaire a été observé dans le cas d'une journaliste agissant avec une certaine liberté dans l'exécution de son travail, mais considérant qu'elle se trouvait dans une relation de subordination du fait de la présence de certaines données factuelles jugées décisives : l'entreprise transmettait des ordres concernant la durée, le lieu et l'heure de diffusion de chaque chronique et reportage, et elle délimitait la zone géographique de ses chroniques. Cependant, la décision rendue a estimé que la façon de réaliser les chroniques était sans importance, considérant que tout journaliste bénéficie d'une grande liberté ${ }^{20}$.

C'est pourquoi, lorsque ces nouvelles formes d'organisation du travail apparaissent, dans le cadre de mutations technologiques et de formes de production de plus en plus flexibles, les instances judiciaires s'adaptent facilement, en faisant une lecture souple et pragmatique de la subordination.

\section{A - LE JEU « UNIDIRECTIONNEL » DE CERTAINS INDICES}

La jurisprudence utilise comme technique pour articuler son argumentation celle des indices lui permettant d'identifier les situations de subordination et de travail pour le compte d'autrui. À ce titre, il est essentiel de ne pas confondre « indices » avec « exigences légales». L'exigence est exclusivement celle de la subordination et, selon les circonstances, certains « indices » peuvent être présents ou non, et permettre par une lecture globale de se prononcer pour ou contre la présence du lien de subordination. C'est précisément ce que nous appelons le jeu « unidirectionnel » des indices : leur présence permet d'affirmer qu'il y a subordination, mais leur absence n'implique pas automatiquement l'exclusion de la subordination.

\section{1 - L'exécution personnelle de la prestation de services}

Le travail subordonné est considéré comme impliquant la fourniture de services " intuitu personae », de sorte qu'il ne peut pas être sous-traité à des tiers. Dans le cadre d'un travail indépendant, il est possible de faire appel à un professionnel spécifique et, par conséquent, d'exiger une prestation de service « intuitu personae »; mais à la différence du contrat de travail, le manquement à cette exigence n'est pas considéré comme une faute

19 STS du 7 octobre 2009, rec. 4169/2008, ECLI:ES:TS:2009:6415; STS du 18 mars 2009, rec. 1709/2007; STS du 29 novembre 2010, rec. 253/2010, ECLI:ES:TS:2010/7764.

20 STS du 11 mai 2009, rec. 3704/2007, ECLI:ES:TS:2009:3784. 
inexcusable. Toutefois, dans le cadre d'un contrat de travail, l'« intuitu personae » ne peut être exigé comme condition essentielle du contrat (puisqu'il peut y avoir des exceptions) qu'à la condition que d'autres indices permettent de conclure à un lien de subordination. Ainsi, la jurisprudence considère que la substitution sporadique n'implique pas l'absence de relation de travail, par exemple dans des cas liés à des activités de nettoyage de bureaux ou d'immeubles de copropriété ${ }^{1}$, voire même à l'égard de certains professionnels effectuant un travail hautement technique lorsqu'ils sont remplacés, en périodes de vacances scolaires par exemple ${ }^{22}$.

Ce type de substitution pourrait poser des difficultés si elle intervenait de manière récurrente. C'est une tendance que l'on observe dans certains travaux de distribution de marchandises, lorsque l'ouvrier vient accompagné d'un parent par exemple : I'un est à l'avant du véhicule, tandis que l'autre est assigné aux tâches de réception et de livraison de la marchandise. Tant que la justice n'a pas tranché sur ce type de pratiques, cellesci ne devraient pas être automatiquement exclues du champ d'application du travail subordonné. Il existe, au sein de notre législation du travail, une vieille disposition relative au statut d'auxiliaire associé qui pourrait s'appliquer en l'espèce : « Si le travailleur, selon ce qui a été convenu par écrit, associe un auxiliaire ou un assistant à son travail, l'employeur du premier sera également l'employeur du second » (article 10, paragraphe 2 du Statut des travailleurs).

\section{2 - L'exécution obligatoire des ordres reçus}

L'une des caractéristiques traditionnelles du travail subordonné réside dans l'obligation du travailleur d'exécuter les tâches qui lui sont confiées par l'employeur, celui-ci manifestant ainsi son pouvoir de direction. Inversement, lorsque le prestataire de services est libre de refuser en tout temps une mission qui lui est proposée, on reconnaît là la marque du travail indépendant.

Nonobstant ce qui précède, il peut arriver que la présence d'une clause du contrat accordant au prestataire de services une telle liberté de refus n'entraîne pas automatiquement l'exclusion de la relation de travail. Dans certains cas spécifiques, la jurisprudence, fondée sur la présence d'autres indices de subordination, conclut que dans les faits le travailleur ne jouit pas d'une réelle liberté de refus, puisqu'il est conscient qu'un ou plusieurs refus entraîneront la perte de son emploi. II s'agit de cas de travail irrégulier, où aucun engagement n'est pris par l'entreprise, ce qui pourrait se traduire en pratique comme étant un contrat zéro heure dans la mesure où aucune garantie minimale de travail n'est convenue entre les parties. Dans ce cas précis, le Tribunal suprême opte pour le caractère professionnel de la relation de travail, compte tenu du fait que la liberté d'accepter ou de refuser les ordres est somme toute relative ${ }^{23}$. Cette dernière considération peut être particulièrement pertinente pour évaluer d'autres cas de figure, caractérisés par cette supposée liberté d'acceptation des commandes, comme c'est le cas dans l'économie uberisée.

21 STS du 16 novembre 2017, rec. 2806/2017, ECLI:ES:TS:2017:4552; STS du 20 juillet 2010, rec. 3344/2009, ECLI:ES:TS:2010:4450 ; STS du 25 janvier 2000, rec. 582/1999.

22 STS du 18 mars 2009, rec. 1709/2007.

23 STS du 16 novembre 2017, rec. 2806/2015, ECLI:ES:TS:2017:4552. 


\section{3 - L' « unidirectionnalité » d'autres indices}

Pour qualifier une relation de relation professionnelle, la jurisprudence utilise aussi souvent comme indice le fait que le travail soit effectué sur le lieu de travail de l'employeur. Mais, il suffit de rappeler que le développement du télétravail, expressément admis en tant que forme de contrat de travail (article 13 du Statut des travailleurs), montre que la nonexécution de services sur le lieu de travail ne traduit en aucun cas le caractère autonome de la relation de travail. Pour cette raison, toutes les formes de travail sur les plateformes qui permettent le travail à distance ne sont pas « en soi » un signe d'autonomie des travailleurs.

La contrainte horaire constitue elle aussi un indice traditionnel, permettant de qualifier une relation de travail, mais l'absence de contrainte horaire ne permet pas quant à elle de conclure à l'absence de relation de travail. La généralisation de la flexibilité des horaires de travail, couplée à des formes de travail par objectifs ou par tâches rémunérées, donne lieu à de nouvelles formes d'emploi subordonné. Ce qui compte pour l'employeur, c'est la capacité de contrôler la manière dont le travail est effectué et le résultat, de sorte que la subordination s'exprime au travers du pouvoir organisationnel de l'employeur.

La livraison par le travailleur des produits finis ou des services fournis à l'employeur est également un indice. Mais encore une fois, il s'agit d'un indice " unidirectionnel » : il suffit d'avoir à l'esprit que parfois le travailleur est responsable de la livraison du produit fini et, surtout, du service rendu directement au client ; il le fait directement même si, juridiquement, il le fait par l'intermédiaire de l'employeur.

De même, le choix de la clientèle par l'employeur est généralement un indice pertinent permettant de conclure à une relation de travail, mais dans certaines activités, cet indice peut ne pas être présent sans que cela remette en cause le caractère professionnel de la relation. Par exemple, des cas où le travailleur est tenu d'effectuer un travail de commercial, avec comme mission précisément de chercher des clients pour l'entreprise.

\section{B - LA PERTINENCE DE NOUVEAUX INDICES}

Face à l'émergence de nouvelles formes d'emploi, il convient d'examiner s'il est possible de détecter l'émergence de nouveaux indices qui, sans remplacer ceux existants, auraient un rôle complémentaire à jouer pour détecter, dans des cas spécifiques, la présence de l'exigence de subordination. De façon schématique, il convient de souligner comment, dans le cadre des changements technologiques et organisationnels qui ont lieu, et parallèlement à la perte d'importance de certains aspects traditionnels de la subordination, d'autres changements apparaissent et montrent, de manière tout aussi décisive, que l'employeur conserve le contrôle de l'organisation du travail, élément clé de la subordination.

Dans ce nouveau contexte de numérisation, le fait pour l'employeur d'exiger que le travail soit effectué à l'aide de certains logiciels, habituellement fournis par l'entreprise ellemême, peut devenir un élément de plus en plus pertinent. L'utilisation de ces programmes spécifiques détermine indirectement le mode d'exécution du travail, contient implicitement une multitude d'ordres et d'instructions précises concernant la prestation de service, et devient le mode d'exercice du pouvoir de gestion de l'employeur.

En outre, les technologies de l'information et de la communication appliquées au travail renforcent, avec une intensité inédite jusqu'ici, la capacité de contrôle du travail en temps réel, tant au niveau des délais qu'en termes de méthode et de résultat. Ainsi, la capacité de l'employeur à surveiller étroitement l'évolution du travail peut être un élément 
clé pour conclure à la subordination du travailleur. La subordination a presque toujours été exclusivement liée à l'exercice du pouvoir organisationnel, alors qu'aujourd'hui, la dimension clé de la subordination réside dans le pouvoir de contrôle du salarié ; un contrôle qui s'exerce grâce à des instruments bien plus performants que par le passé.

L'exclusivité dans la prestation de travail ne permet pas de conclure à l'existence d'une relation de travail. Au contraire, le postulat de départ consiste dans la liberté du salarié de travailler pour plusieurs employeurs. Ainsi, l'exclusivité n'est en aucun cas présumée et doit être acceptée par un accord explicite (article 21, paragraphe 1, du Statut des travailleurs). Néanmoins, il est habituel que, chez le travailleur salarié, le travail s'effectue à temps plein, en raison des engagements indirects de pleine disponibilité pour les tâches confiées par l'employeur, tandis que l'exclusivité reste exceptionnelle dans le travail indépendant. Comme nous parlons d'indices factuels et complémentaires dans une vision d'ensemble, il faut considérer que, dès lors qu'une situation génère des exigences matérielles de pleine disponibilité, ces dernières peuvent être prises en considération pour tenter d'identifier la présence ou non de subordination.

Enfin, il peut être pertinent d'observer le traitement réservé par l'entreprise à d'autres travailleurs qui exécutent en son sein des tâches et missions similaires. Lorsque d'autres travailleurs effectuent le même travail, dans des conditions et circonstances identiques, avec un contrat de travail, on peut supposer que d'autres professionnels dans la même situation mais pour lesquels l'employeur a signé un contrat civil ou commercial bénéficieront des mêmes conditions. Le Tribunal suprême a suivi ce raisonnement dans des décisions récentes ${ }^{24}$.

\section{III - L'ESPACE CONSACRÉ AU TRAVAIL AUTONOME ÉCONOMIQUEMENT DÉPENDANT}

Enfin, le rôle joué par le statut de travailleurs autonomes économiquement dépendants, introduit dans notre système juridique en 2007 par le Statut du travailleur autonome, semble déterminant dans ce nouveau contexte, même si, dans les faits, il est encore très peu utilisé. Ceci, notamment en raison des répercussions potentielles sur l'emploi numérique, appelle un débat sur la nécessaire réforme du système juridique des travailleurs autonomes économiquement dépendants, compte tenu des liens étroits qui existent entre les différentes formes de contrats de prestations de services et des similitudes en termes de coûts.

Dans ce domaine, on a constaté que statistiquement, le nombre de travailleurs autonomes qui exercent leur activité pour un seul client est beaucoup plus élevé que le nombre restreint de travailleurs autonomes économiquement établis comme tels. Le législateur, avec une méfiance excessive, a introduit un nombre considérable d'exigences associées à ce statut, empêchant celui-ci d'offrir aux travailleurs indépendants un minimum de protection face à la relation de dépendance économique dans laquelle ils se trouvent vis-à-vis de la société cliente.

24 STS du 24 janvier 2018, rec. 3394/2015, ECLI:ES:TS:2018:608; STS du 8 février 2018, rec. 3389/2015, ECLI:ES:TS:2018:589. 
Cet écart notable entre la réalité sociologique et statistique d'une part, et la réalité de la condition d'autonome économiquement dépendant d'autre part, conduit à un basculement important de ce dernier vers le statut d'autonome ordinaire. II serait donc souhaitable de procéder à une modification du cadre légal, afin d'éviter cette situation et de renforcer le statut du travailleur autonome économiquement dépendant. 